\title{
An Optical System Defies Conventional Band Theory
}

\section{Squeezed wave functions reshape an open quantum system's bulk-boundary properties and generate a new class of parity-time symmetry.}

\section{By Rachel Berkowitz}

1

uantum systems that obey parity-time symmetry exhibit "exceptional points" within their parameter space. In quantum optical systems, these are points where a light beam's energy gains and losses are perfectly balanced, leading to rich critical phenomena, such as extreme sensitivity to external parameters. Parity-time symmetry can be engineered into systems via designs that ensure a balance of inward and outward energy flows (see Viewpoint: PT Symmetry Goes Quantum). Now, Lei Xiao, at Beijing Computational Science Research Center, and colleagues demonstrate a new way to produce both parity-time symmetry and exceptional points in a quantum-optical circuit. Their study could allow tools for tuning exotic behavior in photonic, phononic, and cold-atom systems [1].

Researchers usually achieve the energy input-output balance necessary to generate parity-time symmetry using systems with spatially periodic structures. The emergence of exceptional

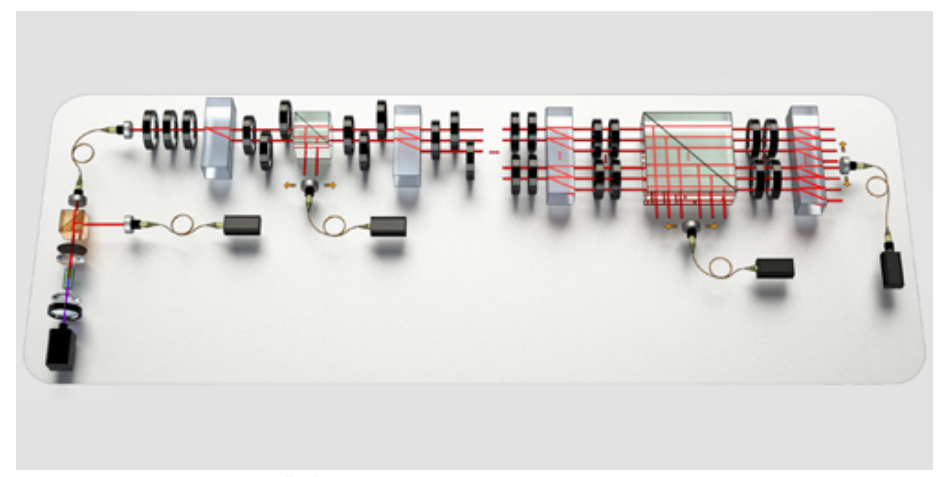

Credit: L. Xiao et al. [1] points in such systems is predicted using Bloch band theory. In their experiment, Xiao and his colleagues observed the presence of parity-time symmetry and exceptional points by measuring how photons interact with themselves as they propagate through a series of optical elements. Although this system has a periodic spatial structure in its bulk, it has an open boundary condition, meaning its properties cannot be described by conventional Bloch band theory.

The researchers concluded that parity-time symmetry arose in their experiment as a result of the "skin effect," meaning that wave functions are squeezed toward a system's boundary. Because it skews the bulk wave functions, the skin effect can only be described by an unconventional "non-Bloch band theory." Designing artificial materials to harness this mechanism could lead to quantum devices with high-resolution sensing capabilities.

Rachel Berkowitz is a Corresponding Editor for Physics based in Vancouver, Canada.

\section{REFERENCES}

1. L. Xiao et al., "Observation of non-Bloch parity-time symmetry and exceptional points," Phys. Rev. Lett. 126, 230402 (2021). 